\title{
cmaJOPEN
}

\section{Effectiveness of ambulation to prevent venous thromboembolism in patients admitted to hospital: a systematic review}

\author{
Brandyn D. Lau MPH, Patrick Murphy MD MPH MS, Anthony J. Nastasi MHS, Stella Seal MLS, \\ Peggy S. Kraus PharmD, Deborah B. Hobson MSN, Dauryne L. Shaffer MSN, Christine G. Holzmueller MS, \\ Jonathan K. Aboagye MBChB MPH, Michael B. Streiff MD, Elliott R. Haut MD PhD
}

\section{Abstract}

Background: Patient ambulation is frequently recommended to help prevent venous thromboembolism during hospital admission. Our objective was to synthesize the evidence for ambulation as a prophylaxis for venous thromboembolism in hospital.

Methods: We conducted a systematic review. We searched MEDLINE, Embase, Scopus, Web of Science and Cochrane Central Register of Controlled Trials indexed from their inception through April 2020 for studies of adult patients admitted to hospital, in which ambulation or mobilization alone or concomitant with prophylaxis was indicated for prevention of venous thromboembolism. We searched ClinicalTrials.gov for unpublished trials. We included randomized controlled trials (RCTs) and observational studies. Two reviewers independently screened articles and assessed risk of bias using 2 validated tools. We scored studies on quality of reporting, internal and external validity and study power; combined scores determined the overall quality.

Results: Eighteen articles met the inclusion criteria: 8 retrospective and 2 prospective cohorts, 7 RCTs and 1 secondary analysis of an RCT. The intervention (ambulation or mobilized) groups varied across studies. Five studies examined exercise as a therapeutic prophylaxis for thrombosis and 9 described an ambulation protocol. Five studies attempted to quantify amount and duration of patient ambulation and 3 reported ambulation distance. In the 5 studies rated as good or excellent statistical quality, findings were mixed. Incidence of venous thromboembolism was lowest when pharmacologic anticoagulants were added as part of the prescribed prophylaxis regimen.

Interpretation: We did not find high-quality evidence supporting ambulation alone as an effective prophylaxis for venous thromboembolism. Ambulation should not be considered an adequate prophylaxis for venous thromboembolism, nor as an adequate reason to discontinue pharmacologic prophylaxis for venous thromboembolism during a patient's hospital admission.

V enous thromboembolism is a global problem, and in the United States alone, it affects up to 600000 patients annually. ${ }^{1}$ Prolonged immobility is a cited risk factor for developing venous thromboembolism. ${ }^{2}$ Randomized controlled trials (RCTs) report significant reductions in venous thromboembolism events among surgical and medical patients who received pharmacological prophylaxis while in hospital. ${ }^{3-5}$ Clinical practice guidelines describe risk-specific recommendations by patient population, ${ }^{6-9}$ and accrediting bodies endorse prevention of venous thromboembolism as a top practice for patient safety. ${ }^{10-13}$

At our hospital, we significantly increased the proportion of patients who were risk assessed for venous thromboembolism and were prescribed risk-appropriate venous thromboembolism prophylaxis, ${ }^{15-18}$ but also found up to $15 \%$ of prescribed doses were not administered to patients admitted to hospital. ${ }^{19,20}$ Upon surveying health care providers on the wards, we discovered that based on the patient's ambulation status, many were presenting prophylaxis doses for venous thromboembolism as optional..$^{21,22}$ Several national and international clinical guidelines recommend ambulation as adequate prophylaxis for venous thromboembolism. ${ }^{79}$ Further, "ambulatory patient" is ubiquitous in electronic medical records as a valid reason for not prescribing prophylaxis for venous thromboembolism. ${ }^{23}$ To our knowledge, evidence supporting such recommendations is not provided. ${ }^{24,25}$

Competing interests: See the end of the article.

This article has been peer reviewed.

Correspondence to: Elliott Haut, ehaut1@jhmi.edu

CMAJ Open 2020. DOI:10.9778/cmajo.20200003 
Our objective was to synthesize the evidence for ambulation as a prophylaxis for venous thromboembolism among patients admitted to hospital.

\section{Methods}

We used the Cocbrane Handbook for Systematic Reviews of Interventions $^{26}$ for guidance in designing and implementing this review.

\section{Data sources and searches}

The systematic review protocol was developed by a multidisciplinary group of clinicians, researchers and quality improvement experts focused on venous thromboembolism prevention (Appendix 1 available at www.cmajopen.ca/content/8/4/ E832/suppl/DC1). A librarian with a master's degree in library sciences (S.S.) helped develop the search strategy and search terms consistent with ambulation in patients admitted to hospital (Appendix 1 and Appendix 2 available at www.cmajopen.ca/content/8/4/E832/suppl/DC1). We searched MEDLINE (1948-Apr. 28, 2020), Embase (1980-Apr. 28, 2020), Scopus (Apr. 28, 2020), Web of Science (Apr. 28, 2020) and the Cochrane Central Register of Controlled Trials (Apr. 28, 2020). We hand searched reference lists from included articles. ClinicalTrials.gov was searched for unpublished RCTs (Aug. 27, 2018).

\section{Study selection}

Two reviewers (P.M. and A.J.N.) independently screened titles, abstracts and full-text articles in duplicate using inclusion and exclusion criteria and resolved any discrepancies through third-party adjudication. We included studies published in English and with adult patients admitted to hospital, in which ambulation was indicated for venous thromboembolism prevention, either as a single mode of prevention or concomitant with prophylaxis. We included all studies of ambulation for prevention of venous thromboembolism even if the ambulation amount was not quantified by time, distance or frequency. Emed and colleagues found severe heterogeneity in definitions of their exposure variable, "immobility," used in studies of thromboprophylaxis among patients admitted to medical wards, re-emphasizing the problem of inconsistency in definitions when performing studies without a standard measure. ${ }^{27} \mathrm{We}$ excluded case-series reports, studies not specifying ambulation and studies done in outpatient, intensive care unit or rehabilitation settings. To be inclusive of all possible evidence regarding the efficacy and effectiveness of ambulation to prevent venous thromboembolism, we included both RCTs and observational studies.

\section{Data extraction}

Using standardized forms, each reviewer (P.M. and A.J.N.) independently extracted data and convened to compare and resolve any discrepancies. Data were extracted in duplicate from included studies for the following variables: country of origin, study design, patient population, participant characteristics (age, sex), interventions, comparisons, outcome and definition of ambulation.

\section{Data analysis}

DistillerSR (Evidence Partners, Ottawa, Canada) was used for screening and data extraction. We planned to conduct a metaanalysis when data were sufficient (from at least 3 RCTs) and studies were sufficiently homogeneous with respect to key variables (population characteristics, study duration and medication dosing).

\section{Assessment of evidence}

Two reviewers independently assessed the quality of the included studies and the risk of bias using the Downs and Black tool ${ }^{28}$ for nonrandomized trials and observational studies recommended by Cochrane (version 5.1). ${ }^{26}$ To maintain consistency of quality assessment, the Downs and Black tool was also used for assessment of RCTs. Each study was scored on the quality of reporting, both external and internal validity, and study power, and the combined scores determined overall quality (scale: poor, $\leq 14$; fair, 15-19; good, 20-25; and excellent, 26-28).

The Agency for Healthcare Research and Quality evidence grading scheme for conducting comparative effectiveness reviews was adapted for use. ${ }^{29}$

The conclusion of this systematic review was based on a combination of both RCTs and other nonrandomized studies, as it is accepted that systematic reviews can be strengthened with observational studies after considering any study limitations. Evidence described as "high strength" probably reflected an actual effect, "moderate strength" indicated that further research may change the result and "low strength" indicated low confidence in an actual effect and that further research would be very likely to change the result. Insufficient evidence meant no evidence or that the body of evidence had unacceptable deficiencies that precluded a conclusion.

\section{Ethics approval}

As this study was solely based on literature, it was not eligible for institutional ethics approval, and none was sought.

\section{Results}

Of 20917 titles identified from the different sources, 6545 duplicates were removed, leaving 14372 articles. After title, abstract and full-text reviews using inclusion criteria, 14354 articles were excluded, leaving 18 articles for analysis (Figure 1). Of the included articles, 2 studies were prospective cohort studies; ${ }^{30,31} 1$ was described as a case-control study, although it was a matched retrospective cohort study; $; 2$ were retrospective cohort studies involving surgical patients; ${ }^{33-39}$ and 7 were RCTs ${ }^{40-46}$ with an additional study of a secondary analysis of the randomized Medical Patients with Enoxaparin (MEDENOX) trial (Table 1). ${ }^{47}$

\section{Study quality and heterogeneity}

The studies varied in definitions of both ambulation and outcome (Table 2). The statistical quality ratings for included studies were poor $(n=3)$, fair $(n=10)$, good $(n=4)$ and excellent 


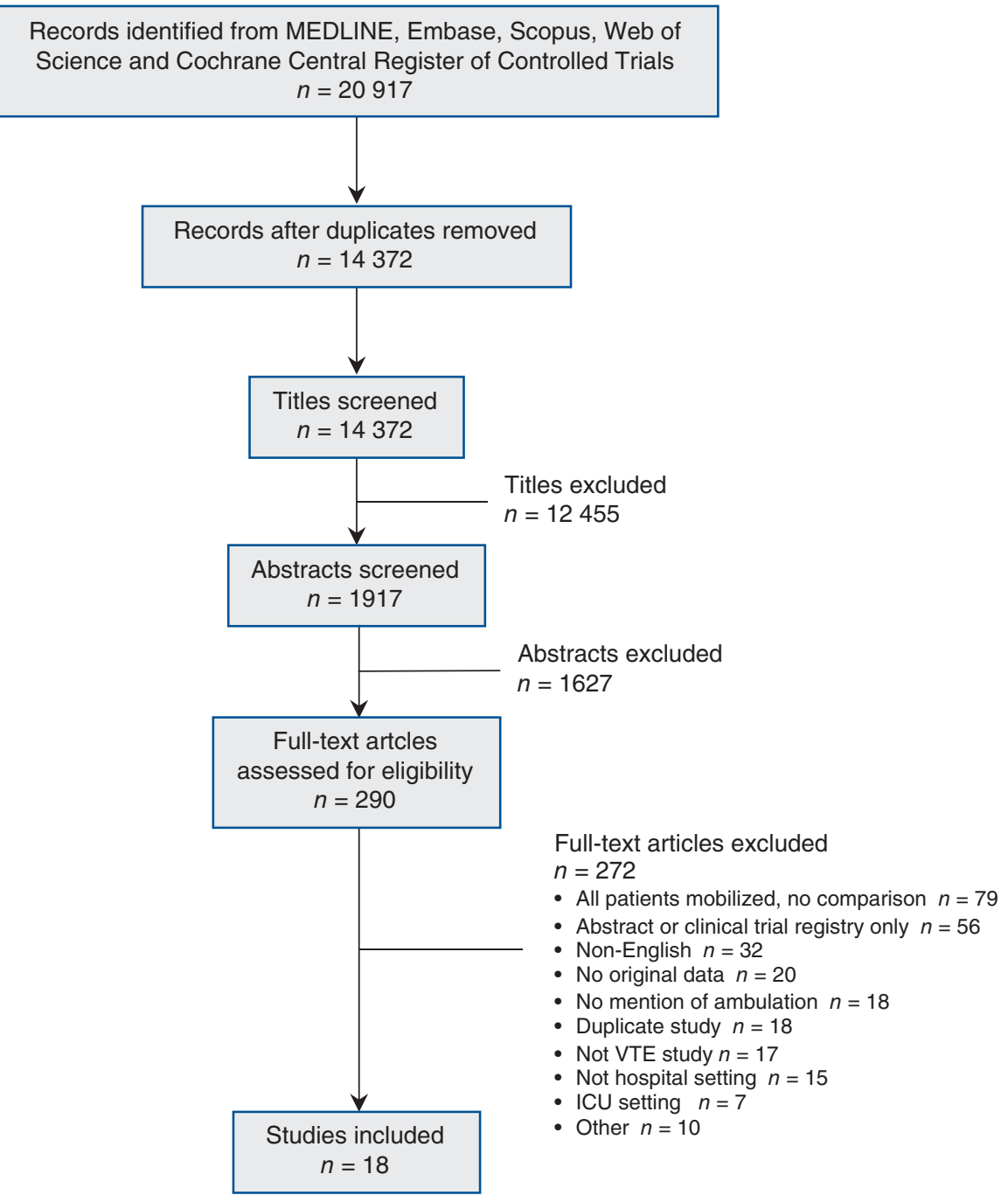

Figure 1: Selection process for studies describing ambulation as a therapy for preventing venous thromboembolism in patients admitted to hospitals. Note: ICU = intensive care unit, VTE = venous thromboembolism.

$(n=1)$ (Table 3)..$^{28}$ Although 3 studies included a power calculation for the primary outcome, only 1 assessed if the sample size was appropriate to detect a clinically meaningful difference in venous thromboembolism, or appropriately powered if no difference was found. ${ }^{46} \mathrm{~A}$ venous thromboembolism event was a secondary outcome in 7 studies. ${ }^{31,32,37,39,42,43,45}$ The use of pharmacologic prophylaxis for venous thromboembolism varied: 6 studies prescribed prophylaxis for all patients, 8 did not report prophylaxis use, 2 did not use prophylaxis and 2 had different regimens by group. The heterogeneity of studies regarding patient populations, pharmacologic prophylaxis and ambulatory interventions precluded the aggregation of data for meta-analysis.

\section{Ambulation definition}

The intervention (ambulation) groups varied across studies. Six studies evaluated exercise (in or out of bed) as a therapeutic prophylaxis for thrombosis and 12 described an ambulatory protocol (Table 2). Five studies attempted to quantify the amount and duration of ambulation ${ }^{31,32,36,45,47}$ and 3 reported the distance of ambulation. ${ }^{36,45,47}$ Amin and colleagues had the most rigorous definition of ambulation (attain autonomous walking distance $>10 \mathrm{~m}$ ), although they did not differentiate by time to achieve this measure. ${ }^{47}$ de Almeida and colleagues quantified ambulation, where ability to walk $3 \mathrm{~m}$ independently was the primary outcome..$^{45}$ Most studies described "early mobilization" or specific prescriptions of mobility, such 


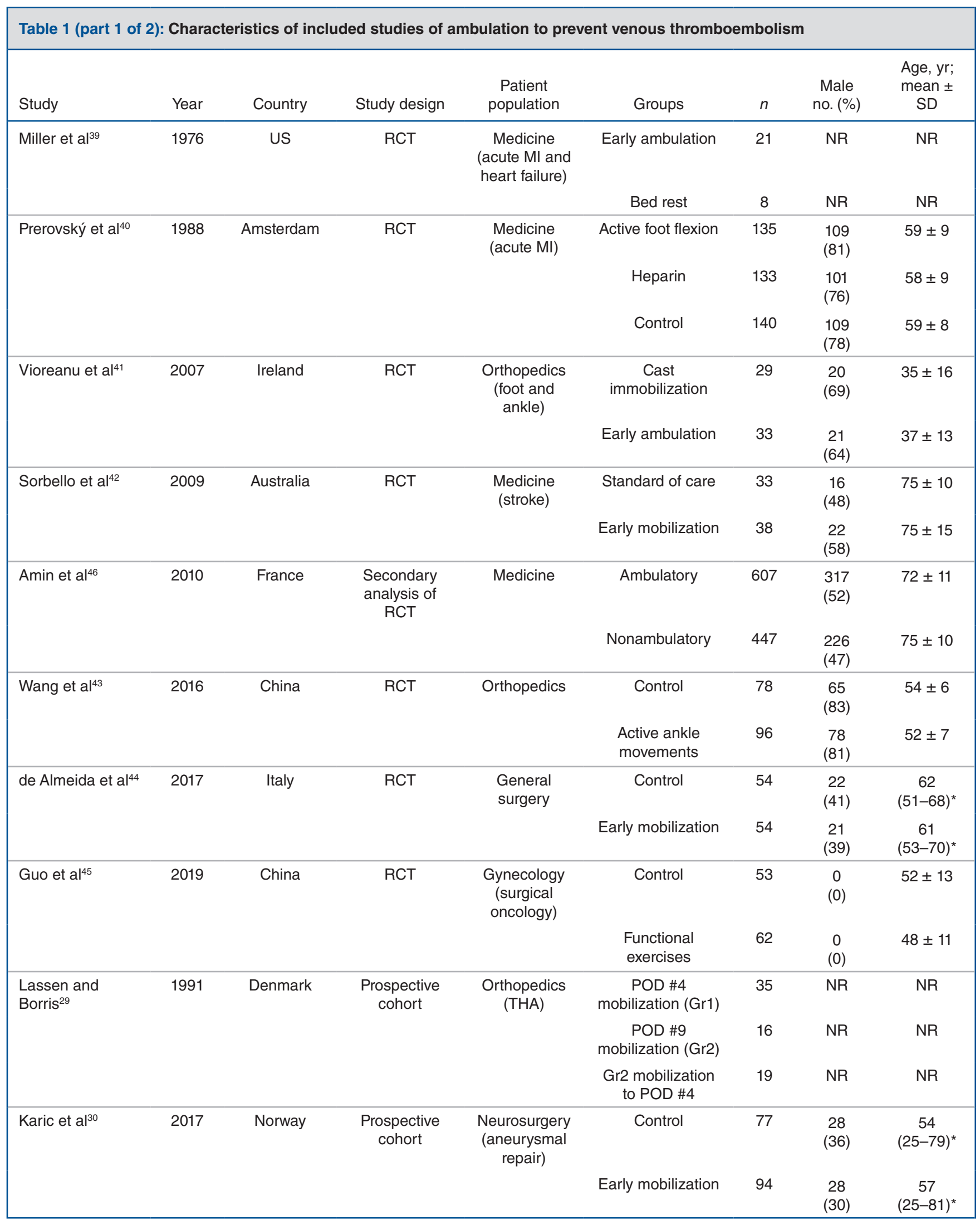




\begin{tabular}{|c|c|c|c|c|c|c|c|c|}
\hline Study & Year & Country & Study design & $\begin{array}{l}\text { Patient } \\
\text { population }\end{array}$ & Groups & $n$ & $\begin{array}{c}\text { Male } \\
\text { no. }(\%)\end{array}$ & $\begin{array}{l}\text { Age, yr; } \\
\text { mean } \pm \\
\text { SD }\end{array}$ \\
\hline \multirow[t]{2}{*}{ Moses $^{32}$} & 1951 & US & $\begin{array}{l}\text { Retrospective } \\
\text { cohort }\end{array}$ & Surgery & Control & 74 & NR & NR \\
\hline & & & & & Bicycle exercise & 74 & NR & NR \\
\hline \multirow[t]{2}{*}{ Flanc et $\mathrm{al}^{33}$} & 1969 & England & $\begin{array}{l}\text { Retrospective } \\
\text { cohort }\end{array}$ & Surgery & Control & 65 & NR & NR \\
\hline & & & & & $\begin{array}{l}\text { Supervised } \\
\text { exercise }\end{array}$ & 67 & NR & NR \\
\hline \multirow[t]{2}{*}{ Pearse et $\mathrm{al}^{34}$} & 2007 & US & $\begin{array}{l}\text { Retrospective } \\
\text { cohort }\end{array}$ & $\begin{array}{l}\text { Orthopedics } \\
\text { (TKA) }\end{array}$ & Early mobilization & 97 & $\begin{array}{c}54 \\
(56)\end{array}$ & $\begin{array}{c}69 \\
(\mathrm{SD} \mathrm{NR})\end{array}$ \\
\hline & & & & & Control & 98 & $\begin{array}{c}48 \\
(49)\end{array}$ & $\begin{array}{c}69 \\
(\mathrm{SD} N R)\end{array}$ \\
\hline \multirow[t]{2}{*}{$\begin{array}{l}\text { Chandrasekaran } \\
\text { et } \mathrm{al}^{35}\end{array}$} & 2009 & Australia & $\begin{array}{l}\text { Retrospective } \\
\text { cohort }\end{array}$ & $\begin{array}{l}\text { Orthopedics } \\
\text { (TKA) }\end{array}$ & $\begin{array}{c}\text { Before ambulation } \\
\text { protocol }\end{array}$ & 50 & $\begin{array}{l}21 \\
(42)\end{array}$ & $\begin{array}{c}73 \\
(\mathrm{SD} N R)\end{array}$ \\
\hline & & & & & $\begin{array}{l}\text { After ambulation } \\
\text { protocol }\end{array}$ & 50 & $\begin{array}{c}24 \\
(48)\end{array}$ & $\begin{array}{c}71 \\
(\mathrm{SD} \mathrm{NR})\end{array}$ \\
\hline \multirow[t]{2}{*}{ Frantzides et al ${ }^{36}$} & 2012 & US & $\begin{array}{l}\text { Retrospective } \\
\text { cohort }\end{array}$ & $\begin{array}{l}\text { General } \\
\text { surgery } \\
\text { (bypass) }\end{array}$ & $\begin{array}{l}\text { Ambulation } \\
\text { protocol }\end{array}$ & 1257 & NR & NR \\
\hline & & & & & Heparin protocol & 435 & NR & NR \\
\hline \multirow[t]{2}{*}{ Cassidy et $\mathrm{al}^{37}$} & 2014 & US & $\begin{array}{c}\text { Retrospective } \\
\text { cohort } \\
\text { (NSQIP) }\end{array}$ & Surgery & $\begin{array}{l}\text { Before VTE } \\
\text { protocol }\end{array}$ & 1569 & NR & NR \\
\hline & & & & & $\begin{array}{l}\text { After VTE QI } \\
\text { protocol }\end{array}$ & 1323 & NR & NR \\
\hline \multirow[t]{2}{*}{ Bhatt et al ${ }^{31}$} & 2017 & Ireland & $\begin{array}{l}\text { Retrospective } \\
\text { cohort }\end{array}$ & $\begin{array}{l}\text { General } \\
\text { surgery }\end{array}$ & Control & 30 & $\begin{array}{c}18 \\
(60)\end{array}$ & $61 \pm 15$ \\
\hline & & & & & Exercise program & 30 & $\begin{array}{c}17 \\
(57)\end{array}$ & $61 \pm 14$ \\
\hline \multirow[t]{2}{*}{ Silver et $\mathrm{al}^{38}$} & 2020 & US & $\begin{array}{l}\text { Retrospective } \\
\text { cohort }\end{array}$ & $\begin{array}{l}\text { Medical } \\
\text { (ischemic } \\
\text { stroke) }\end{array}$ & $\begin{array}{c}\text { Control } \\
\text { (24 h bed rest) }\end{array}$ & 203 & $\begin{array}{c}97 \\
(52)\end{array}$ & $72 \pm 16$ \\
\hline & & & & & $12 \mathrm{~h}$ bed rest & 189 & $\begin{array}{c}87 \\
(46)\end{array}$ & $72 \pm 16$ \\
\hline
\end{tabular}

as twice daily physiotherapy, but failed to report adherence to the defined protocol.

Three studies compared mobility with prolonged immobility. Miller and colleagues compared sitting and standing at the bedside for 30 minutes 3 times a day, starting the first day following myocardial infarction, to 5 days of bed rest, ${ }^{40}$ Lassen and Borris compared mobilization starting on postoperative day 4 to postoperative day $9,{ }^{30}$ and Silver and colleagues compared 12-hour versus 24-hour bed rest following stroke. ${ }^{39}$

\section{Venous thromboembolism diagnosis}

Most studies used clinical suspicion to test for venous thromboembolism, but 8 studies used screening modalities to determine the presence or absence of venous thromboembolism (Table 2). The most common screening modalities were ${ }^{125}$ I-fibrinogen and phlebography. Most studies failed to clarify the diagnostic modality used to confirm the clinical suspicion, particularly studies in which venous thromboembolism was not the primary outcome. Most studies did not report on pulmonary embolism separately.

\section{Ambulation as prophylaxis}

Most studies reported a reduction in venous thromboembolism events with either implementation of an ambulation protocol or promotion of ambulation (Table 2). In the 5 studies rated as good or excellent statistical quality, the findings were mixed 


\begin{tabular}{|c|c|c|c|c|c|c|c|c|c|}
\hline Study & $\begin{array}{l}\text { Ambulatory } \\
\text { group } \\
\text { description }\end{array}$ & $\begin{array}{l}\text { Ambulation } \\
\text { quantified? }\end{array}$ & $\begin{array}{l}\text { Comparison } \\
\text { group } \\
\text { description }\end{array}$ & $\begin{array}{l}\text { Pharmacological } \\
\text { VTE prophylaxis }\end{array}$ & $\begin{array}{l}\text { Outcome } \\
\text { (definition) }\end{array}$ & $\begin{array}{l}\text { Group } \\
\text { sizes }\end{array}$ & \multicolumn{2}{|c|}{ Results } & Study conclusion \\
\hline Miller et $\mathrm{al}^{39}$ & $\begin{array}{l}\text { Sitting and } \\
\text { standing at the } \\
\text { bedside for } 30 \\
\text { min } 3 \text { times/d; } \\
\text { ate meals while } \\
\text { sitting }\end{array}$ & No & $\begin{array}{l}\text { Five days of } \\
\text { bed rest with } \\
\text { leg exercises } \\
\text { hourly }\end{array}$ & No & $\begin{array}{c}\text { DVT } \\
\text { (125/-fibrinogen) }\end{array}$ & $\begin{array}{l}21 \\
8\end{array}$ & $\begin{array}{l}\text { Amb } \\
\text { Control }\end{array}$ & $\begin{array}{l}10 \% \\
63 \%\end{array}$ & $\begin{array}{l}\text { Early mobilization } \\
\text { program reduces } \\
\text { the incidence of } \\
\text { venous thrombosis } \\
\text { in acute Ml }\end{array}$ \\
\hline Prerovský et al ${ }^{40}$ & $\begin{array}{l}\text { Dorsal and } \\
\text { plantar flexion } \\
\text { for } 1-2 \text { min } \\
\text { every hour } \\
\text { while awake }\end{array}$ & No & $\begin{array}{l}\text { Standard of } \\
\text { care without } \\
\text { chemical } \\
\text { VTE } \\
\text { prophylaxis }\end{array}$ & $\mathrm{No}^{*}$ & $\begin{array}{c}\text { DVT } \\
\text { (125/-fibrinogen) }\end{array}$ & $\begin{array}{r}135 \\
133 \\
140\end{array}$ & $\begin{array}{l}\text { Amb } \\
\text { Heparin } \\
\text { Control }\end{array}$ & $\begin{array}{l}5.2 \% \\
9.0 \% \\
13.6 \%\end{array}$ & $\begin{array}{l}\text { Moderate lower } \\
\text { limb exercise is the } \\
\text { simplest measure } \\
\text { to prevent VTE }\end{array}$ \\
\hline Vioreanu et $a^{41}$ & $\begin{array}{l}\text { Custom made } \\
\text { removable } \\
\text { fiberglass cast } \\
\text { with ankle } \\
\text { exercises } \\
3 \text { times } / \mathrm{d} \text { for } \\
10 \mathrm{~min}\end{array}$ & No & $\begin{array}{l}\text { Non- } \\
\text { removable } \\
\text { fiberglass } \\
\text { cast for } \\
6 \text { weeks }\end{array}$ & NR & $\begin{array}{c}\text { VTE } \\
\text { Clinical }\end{array}$ & $\begin{array}{l}29 \\
33\end{array}$ & $\begin{array}{l}\text { Amb } \\
\text { Control }\end{array}$ & $\begin{array}{l}0 \% \\
6 \%\end{array}$ & $\begin{array}{l}\text { Postoperative } \\
\text { immobilization may } \\
\text { increase DVT risk }\end{array}$ \\
\hline Sorbello et al ${ }^{42}$ & $\begin{array}{l}\text { Sitting or } \\
\text { standing within } \\
24 \mathrm{~h} \text { for } 6 \mathrm{~d} \\
\text { with aid of } \\
\text { nurse or } \\
\text { physiotherapist }\end{array}$ & No & $\begin{array}{l}\text { Standard of } \\
\text { care }\end{array}$ & $\mathrm{NR}$ & $\begin{array}{l}\text { VTE } \\
\text { (NR) }\end{array}$ & $\begin{array}{l}33 \\
38\end{array}$ & $\begin{array}{l}\text { Amb } \\
\text { Control }\end{array}$ & $\begin{array}{l}0 \% \\
0 \%\end{array}$ & $\begin{array}{l}\text { No difference in } \\
\text { complications after } \\
\text { initiation of early } \\
\text { mobilization }\end{array}$ \\
\hline Amin et $\mathrm{al}^{46}$ & $\begin{array}{l}\text { Ability to attain } \\
\text { autonomous } \\
\text { walking } \\
\text { distance } \\
>10 \mathrm{~m}\end{array}$ & Yes & $\begin{array}{l}\text { Did not attain } \\
\text { autonomous } \\
\text { walking } \\
>10 \mathrm{~m}\end{array}$ & Yest & VTE (clinical) & $\begin{array}{l}607 \\
447\end{array}$ & $\begin{array}{l}\text { Amb } \\
\text { Control }\end{array}$ & $\begin{array}{l}8.4 \% \ddagger \\
16.2 \%\end{array}$ & $\begin{array}{l}\text { In the prevention of } \\
\text { VTE, reaching } \\
\text { ambulatory status } \\
\text { may not be a reason } \\
\text { for stopping } \\
\text { pharmacological } \\
\text { prophylaxis }\end{array}$ \\
\hline Wang et $\mathrm{a}^{43}$ & $\begin{array}{l}\text { Dorsal and } \\
\text { plantar flexion } \\
30 \text { times/min, } \\
20 \text { times/d in } \\
\text { first } 7 \\
\text { postoperative } \\
\text { days }\end{array}$ & No & $\begin{array}{l}\text { Standard of } \\
\text { care }\end{array}$ & NR & $\begin{array}{l}\text { DVT (doppler } \\
\text { or clinical) }\end{array}$ & $\begin{array}{l}78 \\
96\end{array}$ & $\begin{array}{l}\text { Amb } \\
\text { Control }\end{array}$ & $\begin{array}{l}7.6 \% \\
18.4 \%\end{array}$ & $\begin{array}{l}\text { Significant reduction } \\
\text { in all DVTs but no } \\
\text { difference in } \\
\text { symptomatic DVTs } \\
(2.2 \% \text { v. } 3.9 \%)\end{array}$ \\
\hline $\begin{array}{l}\text { de Almeida et } \\
\mathrm{al}^{44}\end{array}$ & $\begin{array}{l}\text { Twice daily } \\
\text { exercise } \\
\text { program based } \\
\text { on patient's } \\
\text { functional ability }\end{array}$ & Yes & $\begin{array}{l}\text { Once daily } \\
\text { exercise } \\
\text { program }\end{array}$ & NR & DVT (clinical) & $\begin{array}{l}54 \\
54\end{array}$ & $\begin{array}{l}\text { Amb } \\
\text { Control }\end{array}$ & $\begin{array}{l}1.8 \% \\
0 \%\end{array}$ & $\begin{array}{l}\text { Primary outcome } \\
\text { was ability to walk } \\
\text { but no difference in } \\
\text { DVT }\end{array}$ \\
\hline Guo et al ${ }^{45}$ & $\begin{array}{l}\text { Active ankle } \\
\text { motions, calf } \\
\text { massage and } \\
\text { deep breathing }\end{array}$ & No & $\begin{array}{l}\text { Standard of } \\
\text { care }\end{array}$ & Yes & $\begin{array}{l}\text { DVT (clinical or } \\
\text { ultrasonography) }\end{array}$ & $\begin{array}{l}53 \\
62\end{array}$ & $\begin{array}{l}\text { Amb } \\
\text { Control }\end{array}$ & $\begin{array}{l}1.9 \% \\
1.6 \%\end{array}$ & $\begin{array}{l}\text { Because of the } \\
\text { sample size } \\
\text { limitation, the } \\
\text { authors could not } \\
\text { draw any } \\
\text { conclusion about } \\
\text { the effects of } \\
\text { exercise on the } \\
\text { prevention of VTE }\end{array}$ \\
\hline $\begin{array}{l}\text { Lassen and } \\
\text { Borris }^{29}\end{array}$ & $\begin{array}{l}\text { Mobilized from } \\
\text { postoperative } \\
\text { day } 4 \text { onward }\end{array}$ & No & $\begin{array}{l}\text { Mobilized } \\
\text { from } \\
\text { postoperative } \\
\text { day } 9 \text { onward }\end{array}$ & Yes & $\begin{array}{c}\text { DVT } \\
\text { (phlebography) }\end{array}$ & $\begin{array}{l}35 \\
35\end{array}$ & $\begin{array}{l}\text { Amb } \\
\text { Control }\end{array}$ & $\begin{array}{l}21 \% \\
75 \%\end{array}$ & $\begin{array}{l}\text { Patients may lose } \\
\text { benefit of } \\
\text { pharmacological } \\
\text { VTE prophylaxis if } \\
\text { they are not } \\
\text { mobilized }\end{array}$ \\
\hline Karic et $\mathrm{al}^{30}$ & $\begin{array}{l}\text { Progressive } \\
\text { mobilization } \\
\text { from HOB } \\
\text { elevation to } \\
\text { sitting, standing } \\
\text { and walking to } \\
\text { restroom }\end{array}$ & No & $\begin{array}{l}\text { Standard of } \\
\text { care }\end{array}$ & Yes & VTE (clinical) & $\begin{array}{l}77 \\
94\end{array}$ & $\begin{array}{l}\text { Amb } \\
\text { Control }\end{array}$ & $\begin{array}{l}4.2 \% \\
3.8 \%\end{array}$ & $\begin{array}{l}\text { No impact on VTE } \\
\text { but reduced } \\
\text { postoperative } \\
\text { vasospasm }\end{array}$ \\
\hline
\end{tabular}




\begin{tabular}{|c|c|c|c|c|c|c|c|c|c|}
\hline Study & $\begin{array}{l}\text { Ambulatory } \\
\text { group } \\
\text { description }\end{array}$ & $\begin{array}{l}\text { Ambulation } \\
\text { quantified? }\end{array}$ & $\begin{array}{l}\text { Comparison } \\
\text { group } \\
\text { description }\end{array}$ & $\begin{array}{l}\text { Pharmacological } \\
\text { VTE prophylaxis }\end{array}$ & $\begin{array}{l}\text { Outcome } \\
\text { (definition) }\end{array}$ & $\begin{array}{l}\text { Group } \\
\text { sizes }\end{array}$ & \multicolumn{2}{|c|}{ Results } & Study conclusion \\
\hline Moses $^{32}$ & $\begin{array}{l}\text { Forced } \\
\text { respirations } \\
\text { and 2-min } \\
\text { bicycle exercise } \\
\text { every day or } \\
\text { twice daily } \\
\text { while awake }\end{array}$ & No & $\begin{array}{l}\text { Standard of } \\
\text { care }\end{array}$ & NR & VTE (clinical) & $\begin{array}{l}74 \\
74\end{array}$ & $\begin{array}{l}\text { Amb } \\
\text { Control }\end{array}$ & $\begin{array}{l}0 \% \\
5 \%\end{array}$ & $\begin{array}{l}\text { Bicycle or deep } \\
\text { breathing reduce } \\
\text { thrombotic } \\
\text { complications }\end{array}$ \\
\hline Flanc et $\mathrm{al}^{\left.\right|^{33}}$ & $\begin{array}{l}\text { Supervised } \\
\text { exercise } 6 \\
\text { times/d with } \\
\text { nursing } \\
\text { reminders to } \\
\text { exercise }\end{array}$ & No & $\begin{array}{l}\text { Standard of } \\
\text { care }\end{array}$ & NR & $\begin{array}{c}\text { DVT } \\
\text { (125/-fibrinogen) }\end{array}$ & $\begin{array}{l}65 \\
67\end{array}$ & $\begin{array}{l}\text { Amb } \\
\text { Control }\end{array}$ & $\begin{array}{l}25 \% \\
35 \%\end{array}$ & $\begin{array}{l}\text { Strain on hospital } \\
\text { resources and only } \\
\text { benefit was in older } \\
\text { patients }\end{array}$ \\
\hline Pearse et $\mathrm{al}^{34}$ & $\begin{array}{l}\text { VTE prevention } \\
\text { protocol } \\
\text { including }<24 \mathrm{~h} \\
\text { mobilization }\end{array}$ & No & $\begin{array}{l}\text { Routine } \\
\text { ambulation } \\
\text { on POD \#2 }\end{array}$ & Yes & DVT (Doppler) & $\begin{array}{l}97 \\
98\end{array}$ & $\begin{array}{l}\text { Amb } \\
\text { Control }\end{array}$ & $\begin{array}{l}1 \% \\
28 \%\end{array}$ & $\begin{array}{l}\text { Early mobilization } \\
\text { reduces } \\
\text { radiographic DVT }\end{array}$ \\
\hline $\begin{array}{l}\text { Chandrasekaran } \\
\text { et al }{ }^{35}\end{array}$ & $\begin{array}{l}\text { Mobilized with } \\
\text { first } 24 \mathrm{~h} \text {, at } \\
\text { least twice daily, } \\
15-30 \text { min, by } \\
\text { physiotherapists }\end{array}$ & $\begin{array}{l}\text { Yes (sitting, } \\
1-5 \mathrm{~m} \\
>5 \mathrm{~m} \text { ) }\end{array}$ & $\begin{array}{l}\text { Routine out } \\
\text { of bed to } \\
\text { chair and } \\
\text { walking POD } \\
\# 2\end{array}$ & Yes & $\begin{array}{l}\text { VTE (Doppler } \\
\text { or clinical) }\end{array}$ & $\begin{array}{l}50 \\
50\end{array}$ & $\begin{array}{l}\text { Amb } \\
\text { Control }\end{array}$ & $\begin{array}{l}16 \% \\
38 \%\end{array}$ & $\begin{array}{l}\text { Early mobilization } \\
\text { reduces } \\
\text { postoperative DVT, } \\
\text { particularly if > } 5 \mathrm{~m} \\
\text { (no VTE in } 15 \\
\text { patients) }\end{array}$ \\
\hline $\begin{array}{l}\text { Frantzides et } \\
\mathrm{al}^{36}\end{array}$ & $\begin{array}{l}\text { VTE prevention } \\
\text { protocol } \\
\text { including } \\
\text { ambulation } \\
\text { within } 2 \mathrm{~h}\end{array}$ & No & $\begin{array}{l}\text { Standard of } \\
\text { care with } \\
\text { enoxaparin }\end{array}$ & $\begin{array}{c}\text { Yes } \\
\text { (control only) }\end{array}$ & VTE (NR) & $\begin{array}{l}1257 \\
435\end{array}$ & $\begin{array}{l}\text { Amb } \\
\text { Control }\end{array}$ & $\begin{array}{l}0.5 \% \\
2.7 \%\end{array}$ & $\begin{array}{l}\text { Early ambulation } \\
\text { as part of a } \\
\text { comprehensive } \\
\text { protocol obviates } \\
\text { need for } \\
\text { pharmacological } \\
\text { prophylaxis except } \\
\text { in high-risk patients }\end{array}$ \\
\hline Cassidy et $\mathrm{al}^{37}$ & $\begin{array}{l}\text { New } \\
\text { comprehensive } \\
\text { VTE prevention } \\
\text { protocol } \\
\text { including } \\
\text { mobilization } \\
3 \text { times/d }\end{array}$ & No & $\begin{array}{l}\text { Prior to } \\
\text { protocol with } \\
\text { no } \\
\text { predefined } \\
\text { practice }\end{array}$ & $\begin{array}{c}\text { Yes } \\
\text { (according to } \\
\text { risk } \\
\text { assessment) }\end{array}$ & VTE (NSQIP) & $\begin{array}{l}1569 \\
1323\end{array}$ & $\begin{array}{l}\text { Amb } \\
\text { Control }\end{array}$ & $\begin{array}{l}3 \% \\
0.8 \%\end{array}$ & $\begin{array}{l}\text { Postoperative } \\
\text { mobilization } \\
\text { program, risk } \\
\text { stratification and } \\
\text { electronic } \\
\text { recommendations } \\
\text { reduce VTE }\end{array}$ \\
\hline Bhatt et $\mathrm{al}^{31}$ & $\begin{array}{l}\text { Twice daily } \\
\text { exercise } \\
\text { program with } \\
\text { pedal exerciser } \\
\text { or POD\#2 or } \\
\text { when able to sit }\end{array}$ & Yes & $\begin{array}{l}\text { Standard of } \\
\text { care }\end{array}$ & NR & VTE (clinical) & $\begin{array}{l}30 \\
30\end{array}$ & $\begin{array}{l}\text { Amb } \\
\text { Control }\end{array}$ & $\begin{array}{l}0 \% \\
0 \%\end{array}$ & $\begin{array}{l}\text { No impact on VTE } \\
\text { but reduced } \\
\text { infectious } \\
\text { complications } \\
\text { postoperatively }\end{array}$ \\
\hline Silver et al ${ }^{38}$ & $\begin{array}{l}\text { Bedrest } \\
\text { for } \geq 24 \mathrm{~h}\end{array}$ & No & $\begin{array}{l}\text { At least } 12 \mathrm{~h} \\
\text { of bedrest }\end{array}$ & No & DVT (clinical) & $\begin{array}{l}203 \\
189\end{array}$ & $\begin{array}{l}\text { Amb } \\
\text { Control }\end{array}$ & $\begin{array}{l}0.5 \% \\
1.5 \%\end{array}$ & $\begin{array}{l}\text { No effect on VTE but } \\
\text { reduction in } \\
\text { pneumonia and LOS }\end{array}$ \\
\hline
\end{tabular}

(Table 4). Sorbello and colleagues targeted patients admitted to hospital for stroke and found no difference in events among groups (randomized to very early mobilization with physiotherapy v. standard of care). ${ }^{43}$ Cassidy and colleagues conducted a retrospective analysis using the National Surgical Quality Improvement Program database and found a reduction in events from $3 \%$ to $0.8 \%$ after introducing a hospital-wide quality improvement protocol for venous thromboembolism. ${ }^{38}$ The protocol included a standardized risk-stratification protocol combined with a postoperative mobilization program. ${ }^{38}$ This mobilization program required the patient to be out of bed at least 3 times a day starting the day of surgery and "early ambulation" was encouraged.

de Almeida and colleagues compared twice daily graduated exercise protocols to once daily in surgical oncology patients to determine if increased mobility improved functional capacity (ability to walk $3 \mathrm{~m}$ ), and although events were secondary outcomes, no difference was seen. ${ }^{45}$ Guo and colleagues evaluated prescribed "functional exercises" including deep breathing, active ankle mobility and calf 
Table 3: Quality of included studies and assessment of bias, as evaluated by Downs and Black ${ }^{28}$

\begin{tabular}{|c|c|c|c|c|c|c|c|c|}
\hline \multirow[b]{2}{*}{ Study } & \multirow[b]{2}{*}{ Year } & \multirow[b]{2}{*}{ Study design } & \multicolumn{4}{|c|}{ Measure } & \multirow[b]{2}{*}{ Score } & \multirow[b]{2}{*}{$\begin{array}{l}\text { Overall } \\
\text { quality }\end{array}$} \\
\hline & & & $\begin{array}{l}\text { Quality } \\
\text { of } \\
\text { reporting }\end{array}$ & $\begin{array}{l}\text { External } \\
\text { validity }\end{array}$ & $\begin{array}{l}\text { Internal } \\
\text { validity }\end{array}$ & Power & & \\
\hline Moses $^{32}$ & 1951 & Retrospective cohort & 2 & 1 & 1 & 0 & 4 & Poor \\
\hline Flanc et $\mathrm{al}^{33}$ & 1969 & Retrospective cohort & 7 & 2 & 6 & 0 & 14 & Fair \\
\hline Miller et al ${ }^{39}$ & 1976 & RCT & 5 & 1 & 6 & 0 & 12 & Poor \\
\hline Prerovský et al ${ }^{40}$ & 1988 & $\mathrm{RCT}$ & 6 & 2 & 6 & 0 & 14 & Fair \\
\hline Lassen and Borris ${ }^{29}$ & 1991 & Prospective cohort & 3 & 1 & 4 & 0 & 8 & Poor \\
\hline Pearse et $\mathrm{al}^{34}$ & 2007 & Retrospective cohort & 9 & 0 & 6 & 0 & 15 & Fair \\
\hline Vioreanu et al ${ }^{41}$ & 2007 & $\mathrm{RCT}$ & 7 & 3 & 6 & 0 & 16 & Fair \\
\hline Chandrasekaran et al ${ }^{35}$ & 2009 & Retrospective cohort & 8 & 1 & 8 & 0 & 17 & Fair \\
\hline Sorbello et al ${ }^{42}$ & 2009 & $\mathrm{RCT}$ & 10 & 3 & 7 & 0 & 20 & Good \\
\hline Amin et $\mathrm{al}^{46}$ & 2010 & Secondary analysis of RCT & 11 & 3 & 9 & 0 & 23 & Good \\
\hline Frantzides et $\left.a\right|^{36}$ & 2012 & Retrospective cohort & 7 & 3 & 4 & 0 & 14 & Fair \\
\hline Cassidy et al ${ }^{37}$ & 2014 & Retrospective cohort (NSQIP) & 8 & 3 & 8 & 0 & 19 & Good \\
\hline Bhatt et al ${ }^{31}$ & 2017 & Retrospective cohort & 8 & 2 & 6 & 0 & 16 & Fair \\
\hline Wang et $\mathrm{al}^{43}$ & 2016 & $\mathrm{RCT}$ & 8 & 1 & 9 & 0 & 18 & Fair \\
\hline Karic et al ${ }^{30}$ & 2017 & Prospective cohort & 8 & 3 & 6 & 1 & 18 & Fair \\
\hline de Almeida et $\mathrm{al}^{44}$ & 2017 & $\mathrm{RCT}$ & 11 & 3 & 11 & 1 & 26 & Excellent \\
\hline Guo et $\mathrm{al}^{45}$ & 2019 & $\mathrm{RCT}$ & 11 & 2 & 11 & 1 & 25 & Good \\
\hline Silver et $\mathrm{al}^{38}$ & 2019 & Retrospective cohort & 9 & 3 & 5 & 0 & 17 & Fair \\
\hline
\end{tabular}

massage, and although ultrasonography screening was used, there was no difference in the rates of deep venous thrombosis among groups. ${ }^{46}$

Amin and colleagues' study best quantified the actual ambulation of medically ill patients and accurately determined the use of pharmacologic prophylaxis for venous thromboembolism (placebo, enoxaparin $20 \mathrm{mg}$ or enoxaparin $40 \mathrm{mg}$ ) ${ }^{47} \mathrm{In}$ the ambulatory group, with patients achieving autonomous ambulation more than $10 \mathrm{~m}$, the incidence of venous thromboembolism was $8.4 \%$, which was half the incidence of the group not achieving autonomous ambulation. When pharmacologic prophylaxis (enoxaparin $40 \mathrm{mg}$ ) was considered with autonomous ambulation, the incidence of venous thromboembolism was further reduced to $3 \%$. In patients achieving ambulation more than $10 \mathrm{~m}$ independently (but not receiving pharmacologic prophylaxis), the event rate was $10.6 \%$.

Two additional studies at higher risk of bias quantified ambulation or exercise. Bhatt and colleagues did not report any venous thromboembolism events in their study of postoperative use of a pedal exerciser. ${ }^{32}$ Chandrasekaran and colleagues screened all included patients with a duplex ultrasonography on postoperative day 4 and found that patients walking more than $5 \mathrm{~m}$ did not have any deep venous thrombosis or pulmonary embolism compared with the control group (32\% v. $6 \%$ pulmonary embolism, respectively). ${ }^{36}$

\section{Interpretation}

Our systematic review showed a paucity of evidence to support ambulation as an adequate prophylaxis to prevent venous thromboembolism. We found 18 studies over a 69-year span that studied ambulation, most indirectly, to prevent venous thromboembolism; only one-quarter were rated good or excellent quality. We planned a meta-analysis, but the heterogeneity of studies and the varied ambulation definitions made it impossible to quantify any therapeutic ambulation dose.

Four of the 7 RCTs had fewer than 100 patients, and the largest RCT $(n=408)$ defined ambulation as dorsal and plantar flexion for 1 to 2 minutes every hour. ${ }^{41}$ The highest quality study did quantify ambulation and conducted a secondary analysis to compare venous thromboembolism rates with and without pharmacologic prophylaxis. ${ }^{47}$ Although patients achieving autonomous walking in that study had a lower rate of venous thromboembolism events, it is unclear if there were uncontrolled variables to account for this difference. Moreover, the study was designed to investigate the effect of pharmacological prophylaxis on events, which showed a substantial effect in the study. The authors concluded that patients who ambulated more than $10 \mathrm{~m}$ independently and received $40 \mathrm{mg}$ enoxaparin had fewer venous thromboembolism events, 


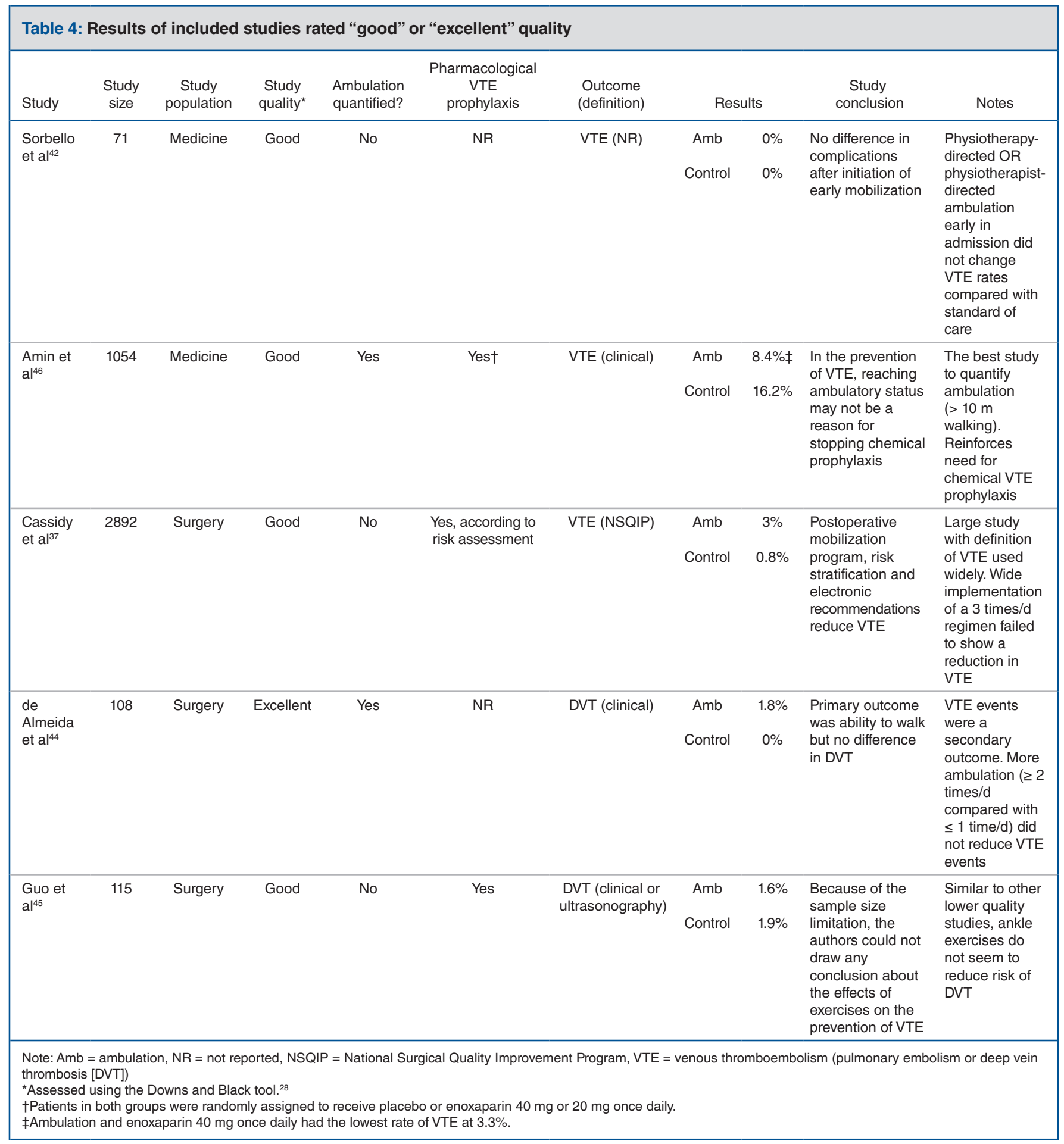

though the incidence was still $3.3 \%$, and the ambulation-only group had an event rate of $10.6 \% .{ }^{47}$ The only other RCT receiving an excellent quality rating primarily investigated the ability to walk after major oncologic resection, but observed no difference in deep venous thrombosis events among groups. ${ }^{45}$

Ambulation or mobilization remains a commonly reported approach, often as the sole prophylaxis, against venous throm- boembolism. Most concerning is that ambulation is often a cited reason to discontinue pharmacologic prophylaxis for venous thromboembolism. At our hospital, nurses and residents perceived that independently ambulating patients did not need pharmacologic prophylaxis for venous thromboembolism. ${ }^{21,22,48}$ However, there is overwhelming evidence supporting pharmacologic prophylaxis in nearly every applicable population admitted to hospital. 
Immobility is a risk factor for development of venous thromboembolism. However, our systematic review found that mobility, ambulation or mobilization have not been shown to reduce VTE events. Many of the major guidelines recommend early ambulation for prevention of venous thromboembolism. For example, the American College of Chest Physicians recommends early ambulation as the only prophylactic measure needed for low-risk nonorthopedic surgical patients, as measured by the Caprini or Rogers risk assessment tool. ${ }^{49}$ Our results challenge early ambulation as appropriate prophylaxis for venous thromboembolism. A patient's risk should be assessed and evidence-based treatment given, considering the risks and benefits. We are not suggesting that ambulation in medical and surgical inpatients is useless. Immobility has many deleterious effects and patients admitted to hospital should be actively encouraged to ambulate. Based on our results, however, we caution the use of ambulation as the sole prophylaxis for prevention of venous thromboembolism or as the impetus to hold pharmacological prophylaxis when ill patients are in a hypercoagulable state.

Although diagnostic and preventive practices for venous thromboembolism have evolved, we searched decades back because the concept of ambulation is long-lived and we needed to find where the concept originated. As early as 1951, Leithauser and colleagues described the "abuse of ambulation." ${ }^{50}$ They suggest, and we agree, that early ambulation is not having the patient "dangle the feet over the edge of the bed or sit in a chair." Several studies in our review described sitting or standing as ambulation. ${ }^{40,43}$ Cassidy and colleagues counted walking to the washroom as 1 of 3 required mobilization events, ${ }^{38}$ and Sorbello and colleagues defined ambulation as sitting or standing within 24 hours. ${ }^{43}$ Rather, the attending physician should prescribe ambulation therapy, including timing, frequency and duration, and the prescribed ambulation should be monitored to ensure the patient undertakes it. The results of our review suggest that such a program has not been studied.

\section{Limitations}

While comprehensive, our review was limited by the quality of the literature. The RCTs were small and often rated as low quality, and most were negative studies. Most studies failed to define the quality and quantity of ambulation. Older studies did not report or did not use pharmacologic prophylaxis for venous thromboembolism. Therefore, the results must be considered in the modern practices of prevention, which include pharmacologic prophylaxis for venous thromboembolism for most patients admitted to hospital.

We considered only studies published in English and only the ClinicalTrials.gov registry for unpublished studies; it is possible that we failed to identify a relevant study.

Further, most studies were eliminated after title or abstract review, and we may have missed studies with ambulation as a secondary intervention or venous thromboembolism as a secondary outcome. Systematic reviews that find little to no evidence are still important to perform and publish. This key step is critical to delineate clear gaps in the published literature for several reasons. First, researchers can plan new studies to address these concerns. ${ }^{51}$ Second, concepts about the robustness of the evidence can be combatted. This is particularly true for ambulation, which is now ubiquitous as a preventive measure for venous thromboembolism despite a lack of evidence. Third, clinical practice might change, in particular using the concepts of implementation or de-implementation science. ${ }^{52,53}$ Although we used a study protocol, it was not registered with PROSPERO (https://www.crd.york.ac.uk/prospero/).

\section{Conclusion}

Our systematic review failed to find high-quality evidence to suggest that ambulation alone is an appropriate or effective prophylaxis for venous thromboembolism. Although some studies suggest that ambulation may reduce venous thromboembolism events among patients admitted to hospital, we could not draw conclusions about how early, how much, how vigorous or how often ambulation should occur to reduce events effectively. In the context of substantial evidence for pharmacologic prophylaxis to prevent venous thromboembolism, ambulation should not be considered an adequate prophylaxis, nor should ambulation be a reason to discontinue pharmacologic prophylaxis during hospital admission. Our findings point to an important function of systematic reviews, which is to evaluate existing evidence. We rigorously evaluated data from studies over a 69-year span and can conclude that research is needed to assess prescribed therapies for ambulation and determine whether any are effective in preventing venous thromboembolism events.

\section{References}

1. Office of the Surgeon General (US); National Heart, Lung, Blood Institute (US). The surgeon general's call to action to prevent deep vein thrombosis and pulmonary embolism. Rockville (MD): Office of the Surgeon General (US); 2008.

2. Anderson FA Jr, Spencer FA. Risk factors for venous thromboembolism. Circulation 2003;107(Suppl 1):I9-16.

3. Samama MM, Cohen AT, Darmon JY, et al. A comparison of enoxaparin with placebo for the prevention of venous thromboembolism in acutely ill medical patients. Prophylaxis in Medical Patients with Enoxaparin Study Group. $N$ Engl 7 Med 1999;341:793-800.

4. Streiff MB, Lau BD. Thromboprophylaxis in nonsurgical patients. Hematology (Am Soc Hematol Educ Program) 2012;2012:631-7.

5. Agnelli G, Bergqvist D, Cohen AT, et al.; PEGASUS investigators. Randomized clinical trial of postoperative fondaparinux versus perioperative dalteparin for prevention of venous thromboembolism in high-risk abdominal surgery. $\mathrm{Br}$ 7 Surg 2005;92:1212-20.

6. Schünemann HJ, Cushman M, Burnett AE, et al. American Society of Hematology 2018 guidelines for management of venous thromboembolism: prophylaxis for hospitalized and nonhospitalized medical patients. Blood Adv 2018; 2:3198-225.

7. Falck-Ytter Y, Francis CW, Johanson NA, et al. Prevention of VTE in orthopedic surgery patients: Antithrombotic Therapy and Prevention of Thrombosis, 9th ed: American College of Chest Physicians Evidence-Based Clinical Practice Guidelines. Chest 2012;141(Suppl):e278S-325S.

8. Anderson DR, Morgano GP, Bennett C, et al. American Society of Hematology 2019 guidelines for management of venous thromboembolism: prevention of venous thromboembolism in surgical hospitalized patients. Blood Adv 2019;3:3898-944.

9. Venous thromboembolism in over 16s: reducing the risk of hospital-acquired deep vein thrombosis or pulmonary embolism. NICE guideline [NG89]. London (UK): National Institute for Health and Care Excellence; 2018.

10. Shekelle PG, Wachter RM, Pronovost PJ, et al. Making health care safer II: an updated critical analysis of the evidence for patient safety practices. Evid Rep Technol Assess (Full Rep) 2013;211:1-945.

11. Shekelle PG, Pronovost PJ, Wachter RM, et al. The top patient safety strategies that can be encouraged for adoption now. Ann Intern Med 2013;158:365-8. 
12. Lau BD, Haut ER. Practices to prevent venous thromboembolism: a brief review. BM7 Qual Saf 2014;23:187-95.

13. Haut ER, Lau BD. Prevention of venous thromboembolism: brief update review. In: Making Health Care Safer II: An Updated Critical Analysis of the Evidence for Patient Safety Practices. Comparative Effectiveness Review No. 211 (Prepared by the Southern California-RAND Evidence-based Practice Center under Contract No. 290-2007-10062-I). AHRQ Publication No. 13-E001-EF. Rockville (MD): Agency for Healthcare Research and Quality; 2013.

14. Streiff MB, Carolan H, Hobson DB, et al. Lessons from the Johns Hopkins multi-disciplinary venous thromboembolism (VTE) prevention collaborative. BM7 2012;344:e3935.

15. Haut ER, Lau BD, Kraenzlin FS, et al. Improved prophylaxis and decreased preventable harm with a mandatory computerized clinical decision support tool for venous thromboembolism (VTE) prophylaxis in trauma patients. Arch Surg 2012;147:901-7.

16. Zeidan AM, Streiff MB, Lau BD, et al. Impact of a venous thromboembolism prophylaxis "smart order set": Improved compliance, fewer events. Am 7 Hematol 2013;88:545-9.

17. Lau BD, Streiff MB, Hobson DB, et al. Beneficial "halo effects" of surgical resident performance feedback. 7 Surg Res 2016;205:179-85.

18. Lau BD, Arnaoutakis GJ, Streiff MB, et al. Individualized performance feedback to surgical residents improves appropriate venous thromboembolism prophylaxis prescription and reduces potentially preventable vte: a prospective cohort study. Ann Surg 2016;264:1181-7.

19. Shermock KM, Lau BD, Haut ER, et al. Patterns of non-administration of ordered doses of venous thromboembolism prophylaxis: implications for novel intervention strategies. PLoS One 2013;8:e66311.

20. Lau BD, Streiff MB, Kraus PS, et al. Missed doses of venous thromboembolism (VTE) prophylaxis at community hospitals: cause for alarm. 7 Gen Intern Med 2018;33:19-20.

21. Elder S, Hobson DB, Rand CS, et al. Hidden barriers to delivery of pharmacological venous thromboembolism prophylaxis: the role of nursing beliefs and practices. 7 Patient Saf 2016;12:63-8

22. Wong A, Kraus PS, Lau BD, et al. Patient preferences regarding pharmacologic venous thromboembolism prophylaxis. F Hosp Med 2015;10:108-11.

23. Kinnier CV, Ju MH, Kmiecik T, et al. Development of a novel composite process measure for venous thromboembolism prophylaxis. Med Care 2016;54:210-7.

24. Lau BD, Streiff MB, Kraus PS, et al. No evidence to support ambulation for reducing postoperative venous thromboembolism. 7 Am Coll Surg 2014; 219:1101-3.

25. Lau BD, Streiff MB, Pronovost PJ, et al. Venous thromboembolism quality measures fail to accurately measure quality. Circulation 2018;137:1278-84.

26. 13.5.2.3. Tools for assessing methodological quality or risk of bias in nonrandomized studies. In: Cochrane Handbook for Systematic Reviews of Interventions. Version 5.1.0. Higgins JPT, Green S, editors. Oxford (UK): Cochrane Collaboration; 2011.

27. Emed JD, Morrison DR, Des Rosiers L, et al. Definition of immobility in studies of thromboprophylaxis in hospitalized medical patients: a systematic review. 7 Vasc Nurs 2010;28:54-66.

28. Downs SH, Black N. The feasibility of creating a checklist for the assessment of the methodological quality both of randomised and non-randomised studies of health care interventions. 7 Epidemiol Community Health 1998;52:377-84.

29. Berkman ND, Lohr KN, Ansari M, et al. Grading the Strength of a Body of Evidence When Assessing Health Care Interventions for the Effective Health Care Program of the Agency for Healthcare Research and Quality: An Update. Methods Guide for Comparative Effectiveness Reviews (Prepared by the RTIUNC Evidence-based Practice Center under Contract No. 290-2007-10056-I). AHRQ Publication No. 13(14)-EHC130-EF. Rockville, MD: Agency forHealthcare Research and Quality. November 2013.www.effectivehealthcare. ahrq.gov/reports/final.cfm (accessed 2020 Nov. 22).

30. Lassen MR, Borris LC. Mobilisation after hip surgery and efficacy of thromboprophylaxis. Lancet 1991;337:618.

31. Karic T, Røe C, Nordenmark TH, et al. Effect of early mobilization and rehabilitation on complications in aneurysmal subarachnoid hemorrhage. 7 Neurosurg 2017;126:518-26.

32. Bhatt NR, Sheridan G, Connolly M, et al. Postoperative exercise training is associated with reduced respiratory infection rates and early discharge: a casecontrol study. Surgeon 2017;15:139-46.

33. Moses C. Bicycle exercises and deep breathing in the prevention of thrombosis. Angiology 1951;2:139-40.

34. Flanc C, Kakkar VV, Clarke MB. Postoperative deep-vein thrombosis. Effect of intensive prophylaxis. Lancet 1969;1:477-8.

35. Pearse EO, Caldwell BF, Lockwood RJ, et al. Early mobilisation after conventional knee replacement may reduce the risk of postoperative venous thromboembolism. F Bone foint Surg Br 2007;89:316-22.

36. Chandrasekaran S, Ariaretnam SK, Tsung J, et al. Early mobilization after total knee replacement reduces the incidence of deep venous thrombosis. ANZ 7 Surg 2009; 79:526-9.

37. Frantzides CT, Welle SN, Ruff TM, et al. Routine anticoagulation for venous thromboembolism prevention following laparoscopic gastric bypass. 7SLS 2012;16:33-7.
38. Cassidy MR, Rosenkranz P, McAneny D. Reducing postoperative venous thromboembolism complications with a standardized risk-stratified prophylaxis protocol and mobilization program. 7 Am Coll Surg 2014;218:1095-104.

39. Silver B, Hamid T, Khan M, et al. 12 versus 24 h bed rest after acute ischemic stroke thrombolysis: a preliminary experience. 7 Neurol Sci 2020;409:116618.

40. Miller RR, Lies JE, Carretta RF, et al. Prevention of lower extremity venous thrombosis by early mobilization. Confirmation in patients with acute myocardial infarction by 125I-fibrinogen uptake and venography. Ann Intern Med 1976;84:700-3.

41. Prerovský I, Niederle P, Simonová J, et al. Deep vein thrombosis and its prevention in patients with acute myocardial infarction. Cor Vasa 1988;30:345-51.

42. Vioreanu M, Dudeney S, Hurson B, et al. Early mobilization in a removable cast compared with immobilization in a cast after operative treatment of ankle fractures: a prospective randomized study. Foot Ankle Int 2007;28:13-9.

43. Sorbello D, Dewey HM, Churilov L, et al. Very early mobilisation and complications in the first 3 months after stroke: further results from phase II of A Very Early Rehabilitation Trial (AVERT). Cerebrovasc Dis 2009;28:378-83.

44. Wang Z, Chen Q, Ye M, et al. Active ankle movement may prevent deep vein thrombosis in patients undergoing lower limb surgery. Ann Vasc Surg 2016; 32:65-72.

45. de Almeida EPM, de Almeida JP, Landoni G, et al. Early mobilization programme improves functional capacity after major abdominal cancer surgery: a randomized controlled trial. Br $\mathcal{F}$ Anaesth 2017;119:900-7.

46. Guo M, Lu L, Sun Y, et al. Comprehensive functional exercises with patient education for the prevention of venous thrombosis after major gynecologic surgery: a randomized controlled study. Thromb Res 2019;178:69-74.

47. Amin AN, Girard F, Samama MM. Does ambulation modify venous thromboembolism risk in acutely ill medical patients? Thromb Haemost 2010;104:955-61.

48. Piechowski KL, Elder S, Efird LE, et al. Prescriber knowledge and attitudes regarding non-administration of prescribed pharmacologic venous thromboembolism prophylaxis. 7 Thromb Thrombolysis 2016;42:463-70.

49. Gould MK, Garcia DA, Wren SM, et al. Prevention of VTE in nonorthopedic surgical patients: antithrombotic therapy and prevention of thrombosis, 9th ed: American College of Chest Physicians Evidence-Based Clinical Practice Guidelines. Chest 2012;141(Suppl):e227S-77S.

50. Leithauser DJ, Saraf L, Smyka S, et al. Prevention of embolic complications from venous thrombosis after surgery; standardized regimen of early ambulation. 7 Am Med Assoc 1951;147:300-3.

51. Fabian TC. Evidence-based medicine in trauma care: whither goest thou? $f$ Trauma 1999;47:225-32.

52. Neuman HB, Kaji AH, Haut ER. Practical guide to implementation science. FAMA Surg 2020 Jan. 29 [Epub ahead of print]. doi: 10.1001/ jamasurgery.2019.5149.

53. Ho VP, Dicker RA, Haut ER; Coalition for National Trauma Research Scientific Advisory Council. Dissemination, implementation, and de-implementation: the trauma perspective. Trauma Surg Acute Care Open 2020;5:e000423.

Competing interests: Elliott Haut, Michael Streiff, Brandyn Lau and Dauryne Shaffer are supported by a grant from the Agency for Health Research and Quality (R18HS027415). Brandyn Lau, Michael Streiff and Elliott Haut are supported by a grant from the Agency for Health Research and Quality (1R01HS024547) entitled "Individualized Performance Feedback on Venous Thromboembolism Prevention Practice," a contract from the Patient-Centered Outcomes Research Institute (PCORI) entitled "Preventing Venous Thromboembolism (VTE): Engaging Patients to Reduce Preventable Harm from Missed/Refused Doses of VTE Prophylaxis," a contract CE-12-11-4489 "Preventing Venous Thromboembolism: Empowering Patients and Enabling PatientCentered Care via Health Information Technology" from PCORI, and a grant from the National Institutes of Health/National Heart, Lung, and Blood Institute (R21HL129028) entitled "Analysis of the Impact of Missed Doses of Venous Thromboembolism Prophylaxis." Brandyn Lau is supported by the Institute for Excellence in Education Berkheimer Faculty Education Scholar Grant and a contract (AD-1306-03980) from the PCORI entitled "Patient Centered Approaches to Collect Sexual Orientation/Gender Identity Information in the Emergency Department." Deborah Hobson has given expert witness testimony in various medical malpractice cases. Michael Streiff has received research funding from Boehringer-Ingelheim, Janssen, Portola and Roche, consulted for Bayer, CSL Behring, Daiichi-Sankyo, Janssen and Pfizer and has given expert witness testimony in various medical malpractice cases. Elliott Haut is a paid consultant and speaker for the "Preventing Avoidable Venous Thromboembolism - Every Patient, Every Time" VHA/Vizient IMPERATIV Advantage Performance Improvement Collaborative. Elliott Haut receives royalties from Lippincott, Williams, Wilkins for a book - "Avoiding Common ICU Errors." Elliott Haut is a paid consultant and speaker for the Illinois Surgical Quality Improvement Collaborative "ISQIC." Elliott Haut was the paid author of a paper commissioned 
by the National Academies of Medicine, which was used to support the report titled "A National Trauma Care System: Integrating Military and Civilian Trauma Systems to Achieve Zero Preventable Deaths After Injury." No other competing interests were declared.

Affiliations: Russell H. Morgan Department of Radiology and Radiological Science (Lau), Johns Hopkins University School of Medicine; Armstrong Institute for Patient Safety and Quality (Lau, Holzmueller, Streiff), Johns Hopkins Medicine, Baltimore, Md.; Department of Surgery (Murphy), Indiana University, Indianapolis, Ind.; Division of Acute Care Surgery, Medical College of Wisconsin, Wauwatosa, Wis.; London Health Sciences Centre, London, Ont. and Department of Surgery, Indiana University, (Murphy; during the conduct of the study); School of Medicine (Nastasi), Stanford University, Li Ka Shing Building, Stanford, Calif.; Welch Medical Library (Seal), Johns Hopkins University School of Medicine; Department of Pharmacy (Kraus), Johns Hopkins Hospital; Division of Acute Care Surgery (Hobson, Aboagye, Haut), Department of Surgery, School of Medicine, Johns Hopkins University; Department of Nursing (Hobson), Johns Hopkins Hospital; Department of Nursing (Shaffer), Johns Hopkins Hospital; Division of Hematology (Streiff), Department of Medicine, Johns Hopkins University School of Medicine; Department of Health Policy and Management (Haut), Johns Hopkins Bloomberg School of Public Health, Baltimore, Md.
Contributors: Brandyn Lau takes responsibility for the content of the manuscript, including the data and analysis. Brandyn Lau and Patrick Murphy contributed equally to the conception and design, search strategy development, acquisition of data and synthesis, drafting of the manuscript and approval of the final version of the manuscript. Patrick Murphy and Anthony Nastasi screened for relevant articles and scored the quality of included studies. Stella Seal helped develop the search strategy and both Stella Seal and Anthony Nastasi contributed to manuscript revisions and approval of the final version of the manuscript. Peggy Kraus, Deborah Hobson, Dauryne Shaffer, Christine Holzmueller, Jonahthan Aboagye, Michael Streiff and Elliott Haut contributed to conception of study and interpretation of data, revisions of the manuscript and approval of the final version of the manuscript. Elliott Haut provided supervision of the study. Brandyn Lau and Patrick Murphy contributed equally and should be credited as co-first authors. All authors gave final approval of the version to be published and agreed to be accountable for all aspects of the work.

Data sharing: No data will be made available beyond the search strategy, which is described in the appendix.

Supplemental information: For reviewer comments and the original submission of this manuscript, please see www.cmajopen.ca/content/8/4/ E832/suppl/DC1. 